

Gangl, D; Zedler, JA; Włodarczyk, A; Jensen, PE; Purton, S; Robinson, C; (2015) Expression and membrane-targeting of an active plant cytochrome P450 in the chloroplast of the green alga Chlamydomonas reinhardtii. Phytochemistry, 11022 - 28. 10.1016/i.phytochem.2014.12.006. Downloaded from UCL Discovery: http://discovery.ucl.ac.uk/1460689.

\title{
ARTICLE
}

\section{Expression and membrane-targeting of an active plant cytochrome P450 in the chloroplast of the green alga Chlamydomonas reinhardtii}

\author{
Doris Gangl ${ }^{1}$, Julie A. Z. Zedler ${ }^{1}$, Artur Włodarczyk ${ }^{2}$, Poul Erik Jensen ${ }^{2}$, Saul Purton ${ }^{3}$ and \\ Colin Robinson ${ }^{1, *}$ \\ ${ }^{1}$ Centre for Molecular Processing, School of Biosciences, University of Kent, Canterbury \\ CT2 $7 N J$. \\ ${ }^{2}$ Copenhagen Plant Science Centre, Department of Plant and Environmental Sciences, \\ University of Copenhagen, Thorvaldsensvej 40, DK-1871 Frederiksberg C, Copenhagen, \\ Denmark. \\ ${ }^{3}$ Institute of Structural \& Molecular Biology, University College London, Gower Street, \\ London WC1E 6BT. \\ "Corresponding author: Colin Robinson; Telephone: +44 1227 823443; E-mail: c.robinson- \\ 504@kent.ac.uk
}

(C) 2014. This manuscript version is made available under the CC-BY-NC-ND 4.0 license http://creativecommons.org/licenses/by-nc-nd/4.0/

\begin{abstract}
The unicellular green alga Chlamydomonas reinhardtii has potential as a cell factory for the production of recombinant proteins and other novel compounds, but mainstream adoption has been hindered by a scarcity of genetic tools and a need to identify products that can be generated in a cost-effective manner. A promising strategy is to use algal chloroplasts as a site for synthesis of high value bioactive compounds such as diterpenoids since these are derived from metabolic building blocks that occur naturally within the organelle. However, synthesis of these complex plant metabolites requires the introduction of membraneassociated enzymes including cytochrome P450 enzymes (P450s). Here, we show that a gene (CYP79A1) encoding a model $\mathrm{P} 450$ can be introduced into the $C$. reinhardtii chloroplast genome using a simple transformation system. The gene is stably expressed and the P450 is efficiently targeted into chloroplast membranes, where it is active and accounts for $0.4 \%$ of total cell protein. These results provide proof of concept for the introduction of diterpenoid synthesis pathways into the chloroplast of Chlamydomonas reinhardtii.
\end{abstract}

Keywords: Chlamydomonas, chloroplast transformation, cytochrome P450, diterpenoids, thylakoid membrane

\section{Introduction}

The green alga Chlamydomonas reinhardtii has served as an important model organism for studies on photosynthesis, chloroplast biology and cell physiology. However, this freshwater alga has also evoked interest as a production platform for recombinant proteins and other 
products; heterologous genes can be expressed in both the nuclear and chloroplast genomes (Purton et al., 2013; Rasala et al., 2014), and the organism grows rapidly and is relatively inexpensive to culture. These factors raise the possibility that cultures can be grown in large-scale photobioreactors, which significantly reduces the risk of contamination and the escape of genetic modified strains to the environment, and makes rapid scale-up possible. In addition, green algae fall into the GRAS (generally regarded as safe) category, potentially eliminating some downstream processing steps associated with transgenically produced therapeutics (Rasala and Mayfield, 2011; Specht et al., 2010).

The chloroplast of $C$. reinhardtii has been a preferred site for the expression of commercially attractive products since it offers a number of advantages compared to the transformation of the nuclear genome. In the chloroplast, high gene expression levels can be achieved and it is possible to target transgenes to defined sites using homologous recombination. In contrast, genes introduced into the nuclear genome integrate randomly and are therefore prone to position effects and RNA silencing. (Day and Goldschmidt-Clermont, 2011).

In recent years several advances have been made in the field of Chlamydomonas chloroplast transformation (Purton et al. 2013). Nevertheless, most transformation protocols still rely on antibiotic resistance for selection. A commonly used selectable marker is the aadA gene (bacterial aminoglycoside 3"-adenyl transferase), which confers resistance to spectinomycin and streptomycin (Goldschmidt-Clermont, 1991). Alternatively, cloned variants of the endogenous ribosomal RNA genes $r r n S$ and $r r n L$ can be used as markers. These carry point mutations rendering the ribosomes insensitive to certain damaging antibiotics (Newman et al., 1990). However, such antibiotic selection is prone to high rates of false positives, and in the latter case results in a mutant ribosome in which translation of a highly expressed transgene is potentially compromised. Furthermore, ensuring that transgenic lines are homoplasmic (i.e. that all $\sim 80$ copies of the polyploid chloroplast genome carry the introduced transgene) requires multiple rounds of single colony isolation under antibiotic selection. A more elegant method is to rely on the restoration of photosynthetic growth using a Chlamydomonas chloroplast mutant defective in an essential photosynthesis gene such as atpB, or psbH (Boynton et al., 1998; Economou et al. 2014). Importantly, the gene of interest remains the only segment of foreign DNA in the transformed genome. A similar strategy has been described earlier by Cheng et al. (2005).

To-date, over 50 different recombinant proteins have been synthesized successfully in the C. reinhardtii chloroplast, including markers, reporters, enzymes, and proteins of therapeutic value such as antibodies, hormones and vaccines (Purton 2013). A fully functional human lgG1 monoclonal antibody against anthrax protective antigen 83 was expressed from two separate genes and assembled into a fully active antibody (Tran et al., 2009). In addition, immunotoxins for cancer therapeutics (Tran et al., 2013) as well as vaccine candidates against malaria or foot-and-mouth-disease virus have been produced in the chloroplast (Gregory et al., 2013; Jones et al., 2013). However, most of the recombinant proteins produced, have been soluble proteins, and furthermore have generally been proteins that can be considered 'benign' in that they have no effect on chloroplast metabolism. To-date there has been only a few reported attempts to express membrane-associated proteins, or enzymes that introduce novel metabolic pathways (Blatti et al., 2012; Wu et al., 2010).

As a consequence, $C$. reinhardtii has yet to compete effectively with other, heterotrophic production hosts for the production of recombinant biopharmaceuticals. Here, we have taken the approach that algae may be preferentially suited for the production of plant-specific compounds. One such class of compounds is terpenoids. These are large, structurallycomplex plant metabolites that include a range of very high-value examples including paclitaxel, artemisinin and ingenol-3-angelate (Wang, 2005). Importantly, their complex structures almost invariably mean that efficient chemical synthesis is extremely difficult, and usually impossible. In principle, it should be possible to introduce novel terpenoid 
biosynthesis pathways into the plant or algal chloroplast, but this would require re-targeting of the key enzymes in their synthesis, cytochromes P450, into the chloroplasts in an active form. Nielsen et al (2013) have reported the successful synthesis of Dhurrin in tobacco chloroplasts and showed that synthesis is light-driven using native ferredoxin as the electron donor. Dhurrin is a plant defense compound found in Sorghum bicolor. While Dhurrin is not a terpenoid, it is synthesized from tyrosine by the means of three ER localized cytochrome P450 enzymes, CYP79A1 (Sibbesen et al., 1995), CYP71E1 (Kahn et al., 1997) and POR (NADPH cytochrome P450 oxidoreductase), as well as the soluble cytosolic UDP glucosyl transferase UGT85B1 (Jones et al., 1999).

As a first step in introducing in such a pathway into a phototrophic microorganism suitable for industrial cultivation (Specht et al. 2010), we report the stable expression of the cytochrome P450 CYP79A1 in the chloroplast of $C$. reinhardtii using a simple transformation method that results in a transgenic line with the CYP79A1 synthetic gene as the only foreign DNA. The enzyme is targeted into the organelle membrane, accumulates to $\sim 0.4 \%$ of total cellular protein and is shown to be active in the conversion of tyrosine to $p$ hydroxyphenylacetaldoxime. The work paves the way for the introduction of additional components of the Dhurrin pathway.

\section{Results and Discussion}

2.1 Transformation strategy and construction of the chloroplast expression vector

The overall strategy for this study is shown in Figure 1. In the ER, P450s are located in the ER membrane by means of an N-terminal transmembrane span; they drive reactions using reducing power from NADPH but the immediate electron donor is cytochrome P450 reductase (POR). In this study the aim was to express a model P450, CYP79A1, in C. reinhardtii chloroplasts, target the enzyme to the thylakoid membrane and drive the reaction using reduced ferredoxin ( $\mathrm{Fd})$. This has been shown to be possible in transiently transfected tobacco chloroplasts (Nielsen et al., 2013) and our aim was to determine whether algal chloroplasts are a viable production base for stable expression of these enzymes.

A recently-developed method for chloroplast transformation (Economou et al., 2014) uses a $C$. reinhardtii strain in which the chloroplast $p s b H$ gene has been replaced by the aadA antibiotic resistance cassette. The vector pASapl is used to introduce the gene of interest and restore photosynthetic growth. The expression cassette of pASapl is comprised of the promoter, the 5' UTR and the start codon of atpA, a multiple cloning site, and the stop codon and 3'UTR of rbcL. Upon homologous recombination the aadA cassette is replaced with both a functional copy of $p s b H$ and the gene of interest (in this case the coding sequence for CYP79A1; see Figure 2). Importantly, the gene of interest remains the only segment of foreign DNA in the transformed genome.

The CYP79A1 coding sequence was synthesized de novo, incorporating the coding sequence for a C-terminal HA tag to facilitate identification and analysis. The synthesized CYP79A1 construct was cloned into pASapl as indicated in Figure 2, and C. reinhardtii chloroplast transformation was performed by rapid agitation of a DNA/cell mixture with glass beads and selection for photoautotrophic growth. After approximately four weeks transformant colonies were obtained and subjected to further analysis. Control transformations, performed without the addition of DNA, did not yield any colonies.

\subsection{Integration of the CYP79A1 construct into the chloroplast genome}

Correct integration of the CYP79A1 gene into the chloroplast genome was confirmed using a PCR approach. Primers were designed to amplify a $1200 \mathrm{bp}$ fragment from transformed lines (containing an intact restored $\mathrm{psbH}$ gene). Figure 3 shows a PCR analysis of the transgenic line (CYP79A1) with the 1200 bp clearly visible; control PCR reactions using primers for the disrupted psbH gene confirmed the homoplasmic integration of the CYP79A1 construct into the chloroplast of $C$. reinhardtii. 
2.3 Accumulation and localisation of the recombinant CYP79A1 protein in the chloroplast The expression of CYP79A1 was tested by immunoblotting of $C$. reinhardtii samples using antibodies to the HA tag as shown in Figure 4. A clear protein signal of $58 \mathrm{kDa}$, close to the expected size of CYP79A1 (62 kDa) was detected in samples from transgenic cells (CYP79A1 lane) but not in samples from the restored wild type (TN72-RP). The anti-HA antibody also detects a smaller polypeptide, but this is also present in control samples indicating a non-specific reaction (band is denoted n.s.).

To estimate the amount of protein expressed in the chloroplast of Chlamydomonas we used a HA-tagged reference protein, HA-Ubiquitin. Figure 4 shows the signals obtained with increasing loadings of this protein and densitometric analysis of calibrated immunoblots revealed an accumulation of approximately $900 \mathrm{ng}$ of protein per $\mathrm{ml}$ of culture. We calculated that the expressed CYP79A 1 corresponds to $0.4 \%$ of total cell protein.

Since CYP79A1 is a membrane-bound enzyme in its natural environment (the plant ER) we investigated its location in algal cells to confirm membrane targeting and insertion. TN72-RP and CYP79A1 cells were lysed by sonication and the soluble and insoluble fractions were separated by ultracentrifugation. Immunoblotting shows that CYP79A1 signal is almost exclusively detected in the membrane fraction of the transgenic cell line. As a control for the pelleting of thylakoids membranes, we confirmed that essentially all of the chlorophyll (over $98 \%$ ) was pelleted by this procedure (data not shown). (Figure 5). These data show that the CYP79A1 protein is efficiently targeted into membranes during or after synthesis in the chloroplast. The data also show that the band generated by non-specific reaction with the antibody (n.s.) is present in the soluble fraction.

\subsection{Enzymatic activity of CYP79A1}

CYP79A1 is the first enzyme in the dhurrin synthesis pathway and it converts tyrosine to $p$ hydroxyphenylacetaldoxime. To test whether the enzyme is active in the Chlamydomonas transformant chloroplasts, we subjected samples of the transgenic strain and restored wild type to LC-MS analysis. A comparison of the mass spectrum to that of an oxime standard revealed that the enzyme is active in the CYP79A1 transformant (Figure 6); as expected, the restored wild type sample does not exhibit any oxime-related peaks. Interestingly, the oxime was detected in the culture medium of unlysed transformant cells, but was absent in extracts of both the soluble or insoluble fractions after sonication. This shows that the oxime reaction product is able to exit the cells, presumably by diffusion since the cells are unlikely to contain an efflux system for this compound. The much smaller volume of the cells (compared to the culture medium) probably explains why the product is not detected in those samples.

\section{Concluding remarks}

Cytochrome P450 monooxygenases catalyze key reactions in the biosynthetic pathways of many complex natural products including terpenoids. They are normally located in the endoplasmic reticulum by means of $\mathrm{N}$-terminal transmembrane spans (Frear et al., 1969; Zhao et al., 2014). However, these NADPH requiring pathways usually proceed inefficiently, and plants additionally contain numerous other P450s (typically around 300, although not all are expressed at the same time). A given P450 is usually present at relatively low levels, and, in general, In general, any single P450-dependent pathway usually catalyses low-level synthesis of a given product. It is therefore highly attractive to relocate the cytochrome P450-dependent steps from the ER to the chloroplast of $C$. reinhardtii; the key P450s may then be present in higher amounts and able to use reducing power supplied by photosynthesis to drive their reactions. In this proof of concept study we have tested whether CYP79A1, the first enzyme in the biosynthetic pathway of dhurrin, can be expressed in the chloroplast membranes of $C$. reinhardtii in an active form. CYP79A1 was chosen because it is easily assayed and has been transiently expressed in tobacco and targeted to the chloroplast (Nielsen et al., 2013). The enzyme has also been expressed in cyanobacteria 
(Lassen et al, 2014). The results show that the enzyme is indeed expressed, targeted into membranes and active. The protein levels ( $0.4 \%$ of total protein) are sufficiently high to readily detect enzymatic activity, the reaction product being detectable in the culture medium where it is relatively dilute.

There are good reasons to believe that the CYP79A1 is driven directly by reducing power supplied by photosynthesis. P450s do not oxidise NADPH themselves, and in the ER, they are driven by a set of specific enzymes termed NADPH cytochrome P450 oxidoreductase, which transfer reducing power from NADPH.. When CYP79A1 was expressed transiently in tobacco chloroplasts, it was shown to use electrons from photosynthetically reduced ferredoxin (Fd) (Nielsen et al., 2013). It is therefore assumed that the algal-expressed CYP79A1 in this study is similarly driven by reduced Fd, but we should emphasise that this has not been shown directly. The highly negative redox potential of ferredoxin and its high abundance raises the possibility that the cytochrome P450 may work very efficiently in the chloroplast, but we have not compared the specific activities of the ER- and plastid-localised forms and this will be a prioirty for future studies.

While the primary aim of this study was to test the feasibility of expressing cytochrome P450 enzymes in algal chloroplasts, the results have relevance for the wider field of microalgal biotechnology. To date, chloroplast transformation of Chlamydomonas reinhardtii has been used to express soluble proteins such as antibodies or vaccine candidates (Demurtas et al., 2013; Tran et al., 2009), but to our knowledge no membrane-bound protein has been expressed in an active form. Moreover, there are reasons to consider that this may be intrinsically difficult to achieve; the thylakoid and envelope membranes differ radically in lipid composition when compared to other membranes (ca. $80 \%$ galactolipids, whereas the ER is primarily composed of phospholipids). In addition, we assume that the CYP79A1 is targeted into the thylakoid membranewhich accounts for the vast majority of chloroplast membrane surface area (although some CYP79A1 may be targeted to the envelope membranes), and proteins are targeted into this membrane by mechanisms that differ in fundamental respects from those used for insertion of proteins into other membranes, including the ER (Woolhead et al., 2001). It was therefore important to confirm that an ER membrane protein can be targeted into chloroplast membranes at reasonably high levels, in an active form.

To summarise, we here show the first expression of an active, membrane-bound cytochrome P450 in the chloroplast of Chlamydomonas reinhardtii. A simple chloroplast transformation method and selection strategy was used to create a transformant strain that lacks any antibiotic resistance markers. The enzyme almost certainly uses reducing power from photosynthesis to drive the conversion of endogenous tyrosine to an oxime that appears to diffuse from the cell into the medium. This study thus represents a first step in the proof-of-concept for the production of diterpenoids in the Chlamydomonas chloroplast.

\section{Experimental}

Chlamydomonas cultures

Strains were grown in acetate-containing (TAP) medium or minimal (HSM) medium as described in (Harris et al., 2009), however a revised trace metal recipe was used as found in (Kropat et al., 2011). Cells were grown in $100 \mathrm{ml}$ cultures, at $25^{\circ} \mathrm{C}$, shaking at $110 \mathrm{rpm}$ using illumination at $15 \mu \mathrm{mol}$ photons $\mathrm{m}^{-2} \mathrm{~s}^{-1}$.

Plasmid design

The pASapl vector, described in (Economou et al., 2014), was used for the introduction of the CYP79A1 coding sequence into the chloroplast genome. The CYP79A1 amino acid sequence (accession number XP_002466099) was codon optimized using the Codon Usage Optimizer (http://codonusageoptimizer.org/download/) with codon frequency-, pair- and weight tables of highly expressed chloroplast genes handpicked by the developer. The optimized sequence was synthesized using GenScript (Piscataway, USA). The gene was 
cloned into pASapl using the restriction enzymes Sapl and Sphl (New England Biolabs) to create pASapl-CYP79.

Chloroplast transformation

Chloroplast transformation of $C$. reinhardtii was based on a previously described method (Kindle and Sodeinde, 1994; Kindle, 1990) and involved the agitation of an algal/DNA suspension with glass beads of 400-625 $\mu \mathrm{m}$ diameter (Sigma, St Louis, USA). A $400 \mathrm{ml}$ culture grown to early log phase (approx. $2 \times 10^{6} \mathrm{cell} / \mathrm{s} / \mathrm{ml}$ ) was concentrated by centrifugation and resuspended in TAP to $4 \mathrm{ml}$. $300 \mu$ of these cells (at approx. $2 \times 10^{8}$ cells $/ \mathrm{ml}$ ) was added to a sterilised $5 \mathrm{ml}$ test tube containing $300 \mathrm{mg}$ sterile glass beads, followed by $5-10$ $\mu \mathrm{g}$ circular plasmid DNA (either pASapl-CYP or the empty pASapl vector as a control). The mixture was agitated vigorously at the maximum speed of a Vortex Genie II (Fisher, Loughborough, UK) for $15 \mathrm{sec}$. The cells were spread on HSM selective agar plates after mixing with $0.5 \%$ molten $\left(42^{\circ} \mathrm{C}\right)$ agar of the same selective medium. The plates were incubated at $25^{\circ} \mathrm{C}$ covered with tissue paper $\left(\sim 20 \mu \mathrm{mol}\right.$ photons $\left.\mathrm{m}^{-2} \mathrm{~s}^{-1}\right)$ overnight then transferred to a moderate light $\left(\sim 50 \mu \mathrm{mol}\right.$ photons $\left.\mathrm{m}^{-2} \mathrm{~s}^{-1}\right)$ the next day. Transformant colonies were picked after approximately 4 weeks and restreaked from single colonies three times on selective medium to ensure homoplasmicity. The transgenic lines were subjected to PCR analysis to confirm correct integration of the transgene (principle described in Economou et al. 2014). A representative line was chosen and termed TN72-CYP79A1, together with a pASapl transformant as a negative control (= TN72-RP).

Cell disruption

Culture cell pellets were resuspended in sonication buffer $(10 \mathrm{mM}$ Tris- $\mathrm{HCl}$ pH8, $5 \mathrm{mM}$ $\mathrm{MgCl}_{2}$ ) and lysed by sonication. The membrane and soluble fractions were separated by ultracentrifugation (70000 rpm, 1h, $4^{\circ} \mathrm{C}$, TL-100 ultracentrifuge, Beckman, Pasadena, USA).

SDS-PAGE and Immunoblotting

Total chlorophyll content of cultures was determined by extracting chlorophyll with $80 \%$ acetone and measuring absorbance at 645 and $663 \mathrm{~nm}$ (Arnon, 1949). An amount of culture equivalent to $5 \mu \mathrm{g}$ chlorophyll was separated by SDS-PAGE (15\% acrylamide) at $40 \mathrm{~mA}$ using a Bio-Rad Mini-PROTEAN® Tetra cell system. Proteins were blotted onto PVDF (BioTrace $^{\mathrm{TM}}$, Pall Corporation, Port Washington, USA) membranes using a Bio-Rad MiniPROTEAN® Tetra cell system according to manufacturer's instructions. The membranes were blocked overnight with $5 \%$ skimmed milk powder (Thermo Scientific, Waltham, USA) in PBS (Formedium, Norfolk, UK) $+0.01 \%$ Tween-20 (Sigma, St Louis, USA) (PBS-T) at $4^{\circ} \mathrm{C}$, washed with PBS-T and incubated with anti-HA primary (Sigma, St Louis, USA) antibody at a concentration of $1: 1000$ in PBS-T shaking for $1 \mathrm{hr}$ at room temperature. After PBS-T washes they were incubated with an HRP coupled secondary anti-rabbit antibody (1:5000 in PBS-T, Promega) for 1 hour shaking at room temperature. The membranes were washed once again and visualised by enhanced chemiluminescence (Clarity Western ECL Substrate, Bio-Rad) according to manufacturer's instructions. The chemiluminescent signal was recorded using a Bio-Rad ChemiDoc XRS+ system. The exposure time was set to 2 minutes with pictures being taken every 4 seconds. The marker was recorded and overlaid with a selected blot image.

Protein determination

Protein determination was performed using the Bio-Rad DC Protein assay according to manufacturer's instructions. Following cell lysis by sonication and ultracentrifugation, the soluble fraction was taken directly for protein determination. The membrane fraction was first solubilized in $50 \mathrm{mM}$ Tris-acetate $\mathrm{pH}$ 8.2, $2.5 \mathrm{mM}$ EDTA pH 8 and 1\% (w/v) SDS and then subjected to the assay. 
Oxime-extraction and LC-MS analysis

Broken cells were mixed in equal amounts with ethyl acetate and shaken for 5 minutes. The samples were then centrifuged at $10000 \mathrm{x} g$ for 10 minutes, the supernatant was collected and evaporated completely. Media samples were mixed 3:1 with ethyl acetate and shaken for 1 hour. After centrifugation the upper phase was collected and evaporated. The dried samples were resuspended in $80 \% \mathrm{MeOH}$, diluted 4 times with water and subjected to Liquid chromatography-mass spectrometry analysis as described in (Saito et al., 2012). The analysis was carried out using an Agilent 1100 Series LC (Agilent Technologies, Germany) coupled to a Bruker HCT-Ultra ion trap mass spectrometer (Bruker Daltonics, Bremen, Germany). A Zorbax SB-Aq column (Agilent; $3.5 \mu \mathrm{M}, 2.1 \times 150 \mathrm{~mm}$ ) was used at a flow rate of $0.2 \mathrm{~mL} \mathrm{~min}-1$. The oven temperature was maintained at $35^{\circ} \mathrm{C}$. The mobile phases were: $\mathrm{A}, 2 \mathrm{mM}$ ammonium acetate; $\mathrm{B}$, methanol. The gradient program was: 0 to $1 \mathrm{~min}$, isocratic $25 \% \mathrm{~B} ; 1$ to $11 \mathrm{~min}$, linear gradient 25 to $60 \% \mathrm{~B} ; 11$ to $12 \mathrm{~min}$, isocratic $98 \% \mathrm{~B} ; 12$ to $20 \mathrm{~min}$, isocratic $25 \% \mathrm{~B}$. The mass spectrometer was run in positive APCl mode and the recorded mass range was $\mathrm{m} / \mathrm{z}$ 80-350.

\section{Acknowledgements}

The research leading to these results has received funding from the People Programme (Marie Curie Actions) of the European Union's Seventh Framework Programme FP7/20072013/ under REA grant agreement $n^{\circ} 317184$. This material reflects only the author's views and the Union is not liable for any use that may be made of the information contained therein. The authors also gratefully acknowledge financial support (1) from the VILLUM Center of Excellence "Plant Plasticity", (2) from "Plant Power: Light-Driven Synthesis of Complex Terpenoids Using Cytochrome P450s" (12-131834) funded by the Danish Council for Strategic Research, Programme Commission on Strategic Growth Technologies. We thank Carl Erik Olsen for the LC-MS measurements.

The authors declare that there is no conflict of interest associated with this work.

\section{References}

Arnon, D.I. 1949. Copper Enzymes in Isolated Chloroplasts. Polyphenoloxidase in Beta Vulgaris. Plant physiology 24, 1-15.

Blatti, J.L., Beld, J., Behnke, C.A., Mendez, M., Mayfield, S.P. and Burkart, M.D. 2012. Manipulating fatty acid biosynthesis in microalgae for biofuel through protein-protein interactions. PloS one 7, e42949.

Boynton, J.E., Gillham, N.W., Harris, E.H., Hosler, J.P., Johnson, A.M., Jones, A.R., Randolph-Anderson, B.L., Robertson, D., Klein, T.M., Shark, K.B., et al. (1988). Chloroplast transformation in Chlamydomonas with high velocity microprojectiles. Science 240, 1534-1538.Chenga, Q , Day, A., Dowson-Day, M., Shend, G-F. and Dixon, R. (2005). The Klebsiella pneumoniae nitrogenase Fe protein gene (nifH) functionally substitutes for the chlL gene in Chlamydomonas reinhardtii. Bioem. Biophys. Res. Comm. 329, 966-975.

Day, A. and Goldschmidt-Clermont, M. 2011. The chloroplast transformation toolbox: selectable markers and marker removal. Plant biotechnology journal 9, 540-553.

Demurtas, O.C., Massa, S., Ferrante, P., Venuti, A., Franconi, R. and Giuliano, G. 2013. A Chlamydomonas-derived Human Papillomavirus 16 E7 vaccine induces specific tumor protection. PloS one 8, e61473.

Economou, C., Wannathong, T., Szaub, J. and Purton, S. 2014. A Simple, Low-Cost Method for Chloroplast Transformation of the Green Alga Chlamydomonas reinhardtii. Methods Mol Biol 1132, 401-411.

Frear, D.S., Swanson, H.R. and Tanaka, F.S. 1969. N-demethylation of substituted 3(phenyl)-1-methylureas: Isolation and characterization of a microsomal mixed function oxidase from cotton. Phytochemistry 8, 2157-2169. 
Goldschmidt-Clermont, M. 1991. Transgenic expression of aminoglycoside adenine transferase in the chloroplast: a selectable marker of site-directed transformation of chlamydomonas. Nucleic acids research 19, 4083-4089.

Gregory, J.A., Topol, A.B., Doerner, D.Z. and Mayfield, S. 2013. Alga-produced cholera toxin-Pfs25 fusion proteins as oral vaccines. Applied and environmental microbiology 79, 3917-3925.

Harris, E.H., Stern, D.B. and Witman, G.B. 2009. The Chlamydomonas Sourcebook (Second Edition). Oxford:Elsevier Inc.

Jones, C.S., Luong, T., Hannon, M., Tran, M., Gregory, J.A., Shen, Z., Briggs, S.P. and Mayfield, S.P. 2013. Heterologous expression of the C-terminal antigenic domain of the malaria vaccine candidate $\mathrm{Pfs} 48 / 45$ in the green algae Chlamydomonas reinhardtii. Applied microbiology and biotechnology 97, 1987-1995.

Jones, P.R., Moller, B.L. and Hoj, P.B. 1999. The UDP-glucose:p-hydroxymandelonitrile-Oglucosyltransferase that catalyzes the last step in synthesis of the cyanogenic glucoside dhurrin in Sorghum bicolor. Isolation, cloning, heterologous expression, and substrate specificity. The Journal of biological chemistry 274, 35483-35491.

Kahn, R.A., Bak, S., Svendsen, I., Halkier, B.A. and Moller, B.L. 1997. Isolation and reconstitution of cytochrome $\mathrm{P} 450 \mathrm{Ox}$ and in vitro reconstitution of the entire biosynthetic pathway of the cyanogenic glucoside dhurrin from sorghum. Plant physiology 115, 1661-1670.

Kindle, K. and Sodeinde, O. 1994. Nuclear and chloroplast transformation in Chlamydomonas reinhardtii strategies for genetic manipulation and gene expression. Journal of Applied Phycology 6, 231-238.

Kindle, K.L. 1990. High-Frequency Nuclear Transformation of Chlamydomonas reinhardtii. Proceedings of the National Academy of Sciences of the United States of America 87, 1228-1232.

Kropat, J., Hong-Hermesdorf, A., Casero, D., Ent, P., Castruita, M., Pellegrini, M., Merchant, S.S. and Malasarn, D. 2011. A revised mineral nutrient supplement increases biomass and growth rate in Chlamydomonas reinhardtii. The Plant journal : for cell and molecular biology 66, 770-780.

Lassen, L.M, Nielsen, A.Z., Olsen, C.E., Bialek, W., Jensen, K., Møller, B.L. and Jensen, P.E. 2014. PlosOne (in press).

Newman, S.M., Boynton, J.E., Gillham, N.W., Randolph-Anderson, B.L., Johnson, A.M. and Harris, E.H. 1990. Transformation of chloroplast ribosomal RNA genes in Chlamydomonas: molecular and genetic characterization of integration events. Genetics 126, 875-888.

Nielsen, A.Z., Ziersen, B., Jensen, K., Lassen, L.M., Olsen, C.E., Moller, B.L. and Jensen, P.E. 2013. Redirecting Photosynthetic Reducing Power toward Bioactive Natural Product Synthesis. ACS synthetic biology 2, 308-315.

Purton, S., Szaub, J.B., Wannathong, T., Young, R. and Economou, C.K. 2013. Genetic engineering of algal chloroplasts: Progress and prospects. Russian Journal of Plant Physiology 60, 491-499.

Rasala, B.A., Chao, S.S., Pier, M., Barrera, D.J. and Mayfield, S.P. 2014. Enhanced genetic tools for engineering multigene traits into green algae. PloS one 9, e94028.

Rasala, B.A. and Mayfield, S.P. 2011. The microalga Chlamydomonas reinhardtii as a platform for the production of human protein therapeutics. Bioengineered bugs 2, 50 54.

Saito, S., Motawia, M.S., Olsen, C.E., Moller, B.L. and Bak, S. 2012. Biosynthesis of rhodiocyanosides in Lotus japonicus: rhodiocyanoside $A$ is synthesized from (Z)-2methylbutanaloxime via 2-methyl-2-butenenitrile. Phytochemistry 77, 260-267.

Sibbesen, O., Koch, B., Halkier, B.A. and Moller, B.L. 1995. Cytochrome P-450TYR is a multifunctional heme-thiolate enzyme catalyzing the conversion of L-tyrosine to $p$ hydroxyphenylacetaldehyde oxime in the biosynthesis of the cyanogenic glucoside dhurrin in Sorghum bicolor (L.) Moench. The Journal of biological chemistry 270, 3506-3511. 
Specht, E., Miyake-Stoner, S. and Mayfield, S. 2010. Micro-algae come of age as a platform for recombinant protein production. Biotechnology letters 32, 1373-1383.

Tran, M., Van, C., Barrera, D.J., Pettersson, P.L., Peinado, C.D., Bui, J. and Mayfield, S.P. 2013. Production of unique immunotoxin cancer therapeutics in algal chloroplasts. Proceedings of the National Academy of Sciences of the United States of America 110, E15-22.

Tran, M., Zhou, B., Pettersson, P.L., Gonzalez, M.J. and Mayfield, S.P. 2009. Synthesis and assembly of a full-length human monoclonal antibody in algal chloroplasts. Biotechnology and bioengineering 104, 663-673.

Wang, G., Tang, W. and Bidigare R.R. 2005. Terpenoids as therapeutic drugs and pharmaceutical agents. Natural Products 16, 197-225.

Woolhead, C.A., Thompson, S.J., Moore, M., Tissier, C., Mant, A., Rodger, A., Henry, R. and Robinson, C. 2001. Distinct Albino3-dependent and -independent pathways for thylakoid membrane protein insertion. The Journal of biological chemistry 276, 40841-40846.

Wu, S., Huang, R., Xu, L., Yan, G. and Wang, Q. 2010. Improved hydrogen production with expression of hemH and lba genes in chloroplast of Chlamydomonas reinhardtii. Journal of biotechnology 146, 120-125.

Zhao, Y.J., Cheng, Q.Q., Su, P., Chen, X., Wang, X.J., Gao, W. and Huang, L.Q. 2014. Research progress relating to the role of cytochrome P450 in the biosynthesis of terpenoids in medicinal plants. Applied microbiology and biotechnology 98, 23712383.

\section{Figure legends}

Figure 1. Strategy for driving cytochrome P450 activity by photosynthetic electron transport. Left: normal action of P450s. Typical P450s have an $\mathrm{N}$-terminal transmembrane span that anchors the protein in the ER membrane. Reducing power is provided by NADPH via NADPH-cytochrome P450 oxido-reductases (NADPH-P450 ox-red). Right: strategy for driving activity by photosynthetic electron transport: the P450 is synthesised in the chloroplast, targeted into the thylakoid membrane and reducing power is provided by Photosystem I (PSI) via ferredoxin (Fd).

Figure 2. Chloroplast transformation strategy. A: the vector pASapl uses the essential photosynthesis gene $\mathrm{psbH}$ as a selection marker for the targeted integration of a gene of interest (in this case CYP79A1) into the chloroplast genome. The expression cassette is located in the psbH-trnE2 intergenic region and contains the promoter, 5' untranslated region and start codon of atp $A$, a multiple cloning site and the stop codon as well as the 3' untranslated region of $r b c L$. An aadA cassette replaces $p s b H$ in the recipient strain rendering it unable to grow on minimal medium lacking acetate. After transformation a functional copy of $\mathrm{psbH}$ together with the $\mathrm{GOI}$ is introduced back into the genome by homologous recombination. B: pASapl vector design. The pASapl vector was constructed using pUC8 as a backbone. It contains $p s b H$ flanking elements, the promoter and 5 ' untranslated region (UTR) of atpA, a polylinker with Sapl and Sphl sites to introduce the gene of interest and the 3' UTR of rbcL (Economou et al., 2014).

Figure 3. Integration of the CYP79A1 construct. Genomic DNA was isolated from transformed cultures (CYP79A1) and subjected to PCR analysis to test for integration of the CYP79A1 construct. As a control the same was done with untransformed cells (TN72 $\mathrm{psbH}^{-}$) and restored wild type cultures (TN72-RP). The Figure shows the presence of a 1200 bp band in the transgenic lines, which confirms the integration of the expression cassette into the chloroplast genome. Untransformed cells produce a PCR product of $850 \mathrm{bp}$ length.

Figure 4. CYP79A1 is expressed in the chloroplast of $C$. reinhardtii. Total cell protein samples of restored wild type (TN72-RP) and transformant strains (CYP79A1) were 
separated using SDS-PAGE and subjected to immunoblot analysis using an anti-HA antibody. Known amounts of a reference protein (HA-ubiquitin) were loaded on the same gel. CYP79A1 band is indicated; 'n.s.' denotes non-specific cross-reacting band found in both cultures; HA-Ub denotes HA-Ubiquitin.

Figure 5. Membrane targeting of CYP79A1. Cells of the CYP79A1 transformant and restored wild type (TN72-RP) cultures were lysed by sonication and the membrane (M) and soluble (S) fractions were separated by ultracentrifugation. Total protein in the fractions was subjected to SDS-PAGE and immunoblotting with an antibody against the HA-tag. The band corresponding to CYP79A1 is found in the membrane fraction of the transgenic line confirming that CYP79A1 is targeted into the membranes of $C$. reinhardtii. A non-specific cross-reacting band is denoted by n.s.

Figure 6. Expressed CYP79A1 is active. The product of CYP79A1, $p$ hydroxyphenylacetaldoxime, was extracted from the culture medium of CYP79A1 transformant or restored wild type cultures with ethyl acetate after pelleting the cells by centrifugation. The samples were analysed by liquid chromatography-mass spectrometry and compared to an oxime standard as described in the Experimental section. 
A

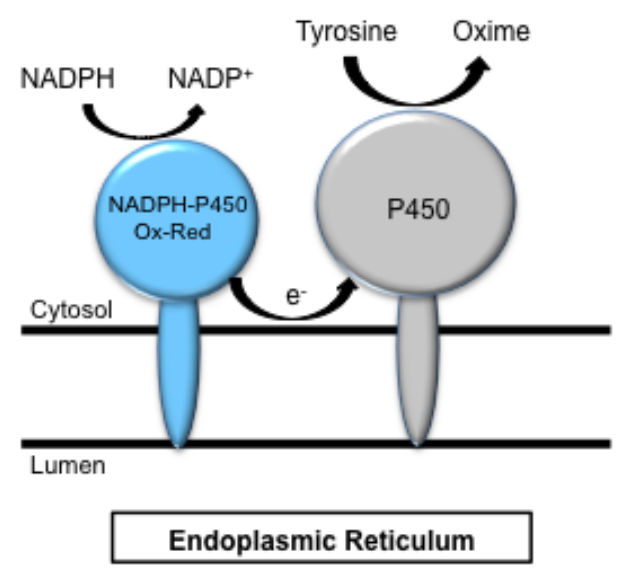

B

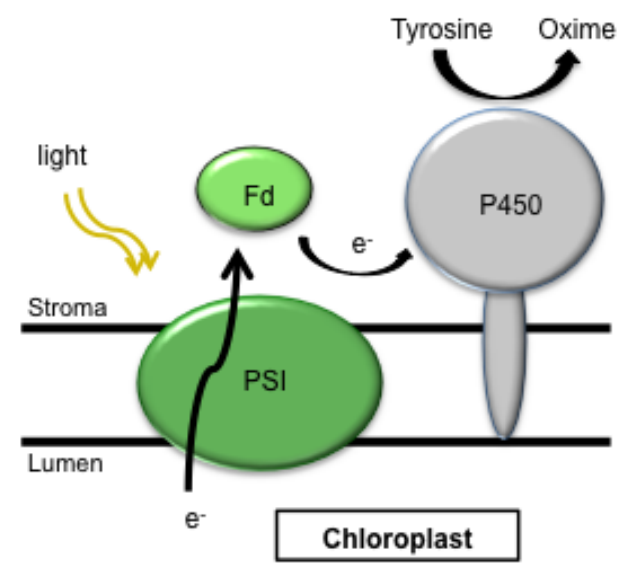

Figure 1 
A
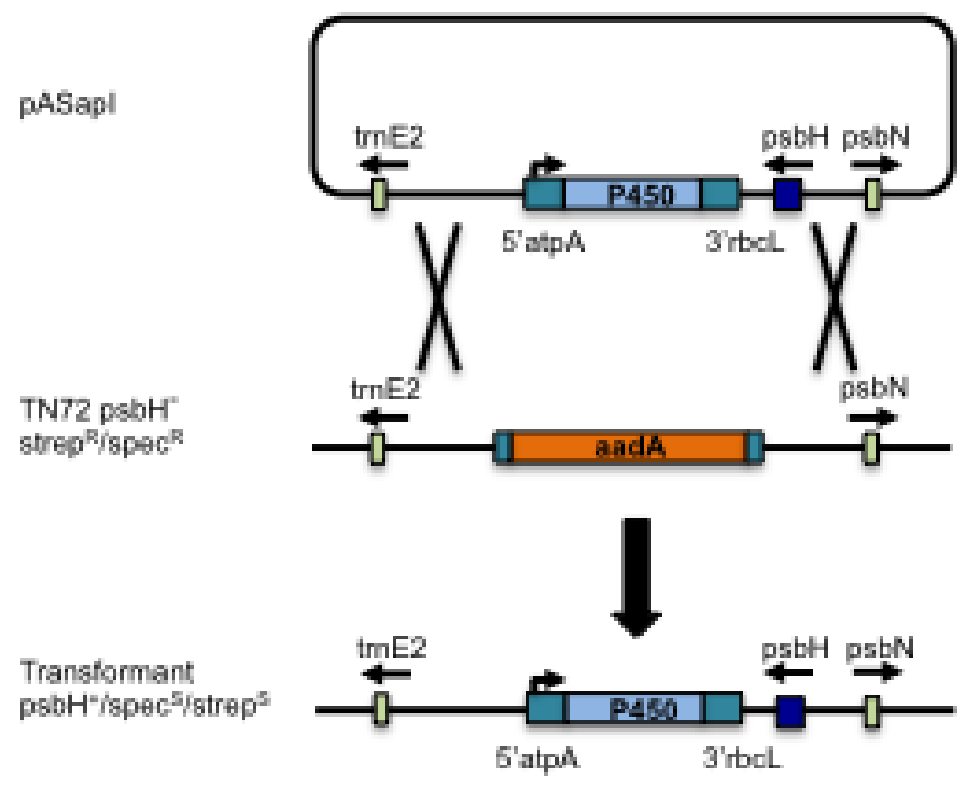

Figure 2

B

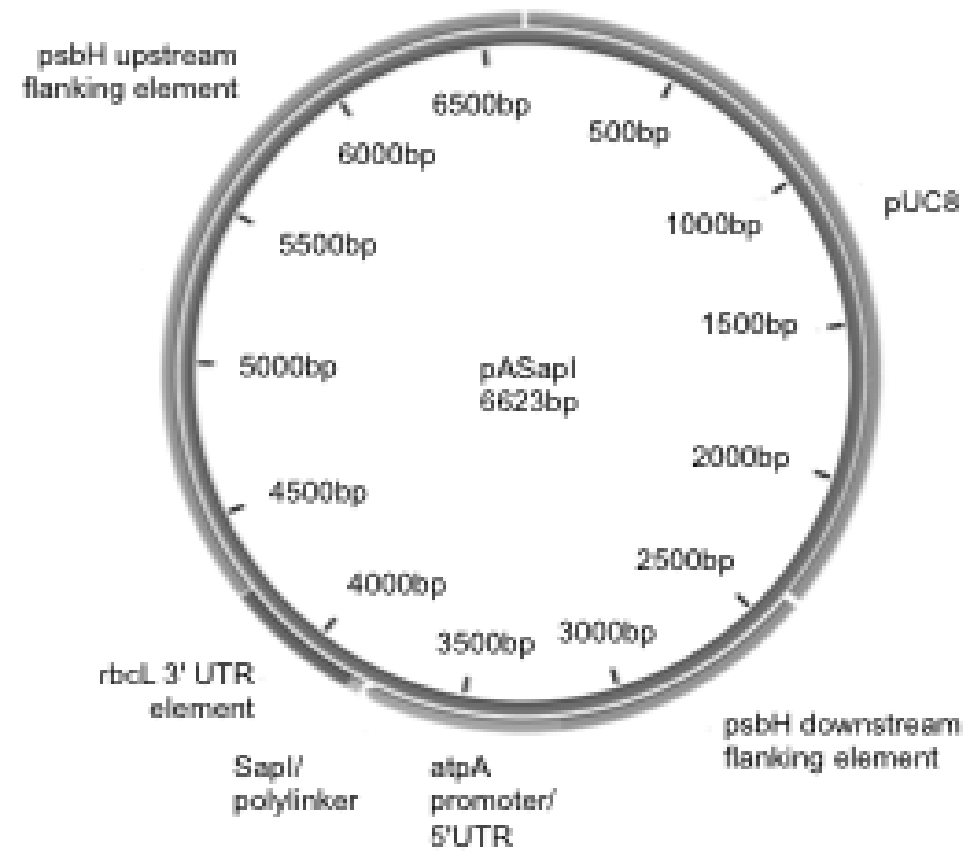




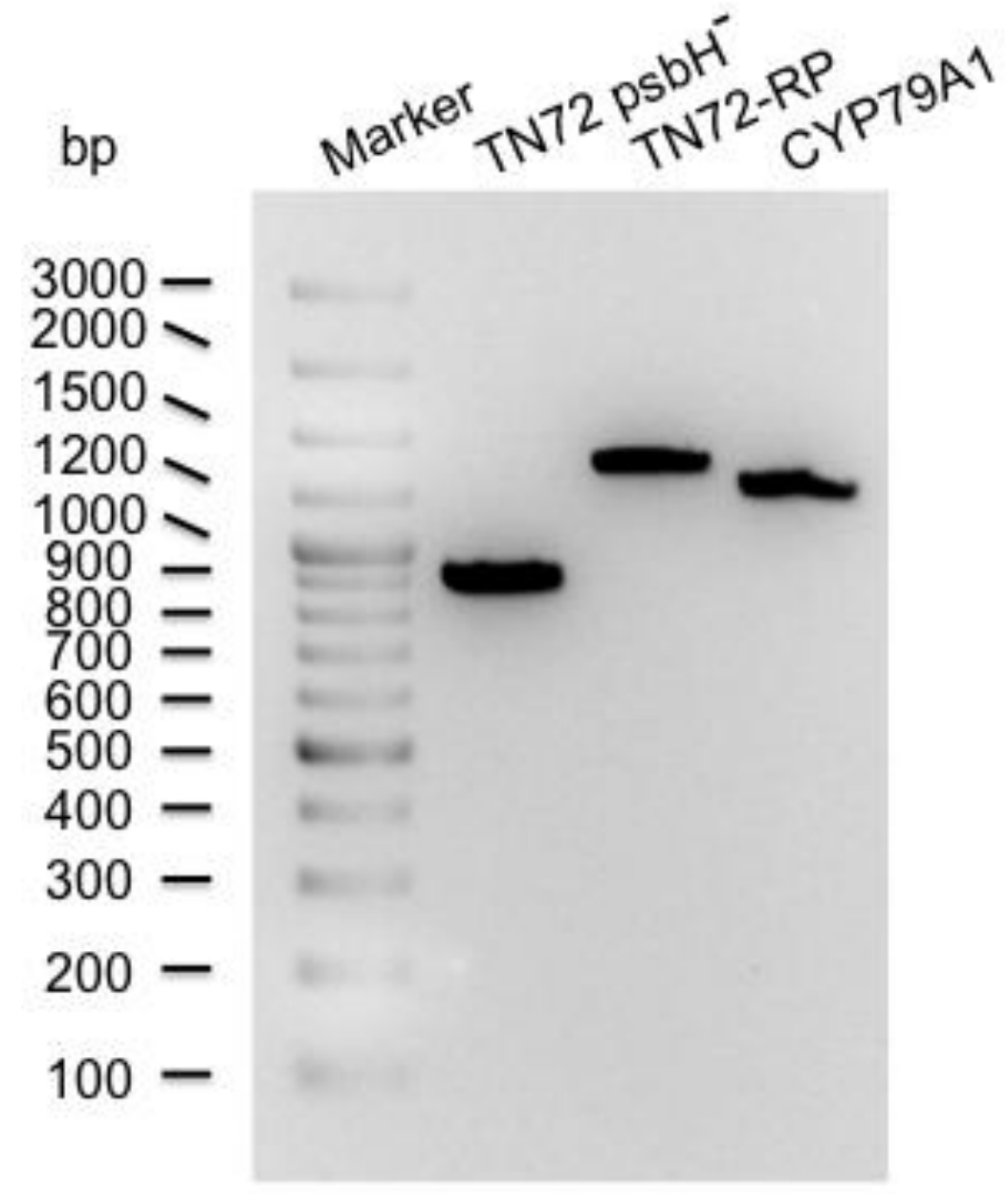

Figure 3 


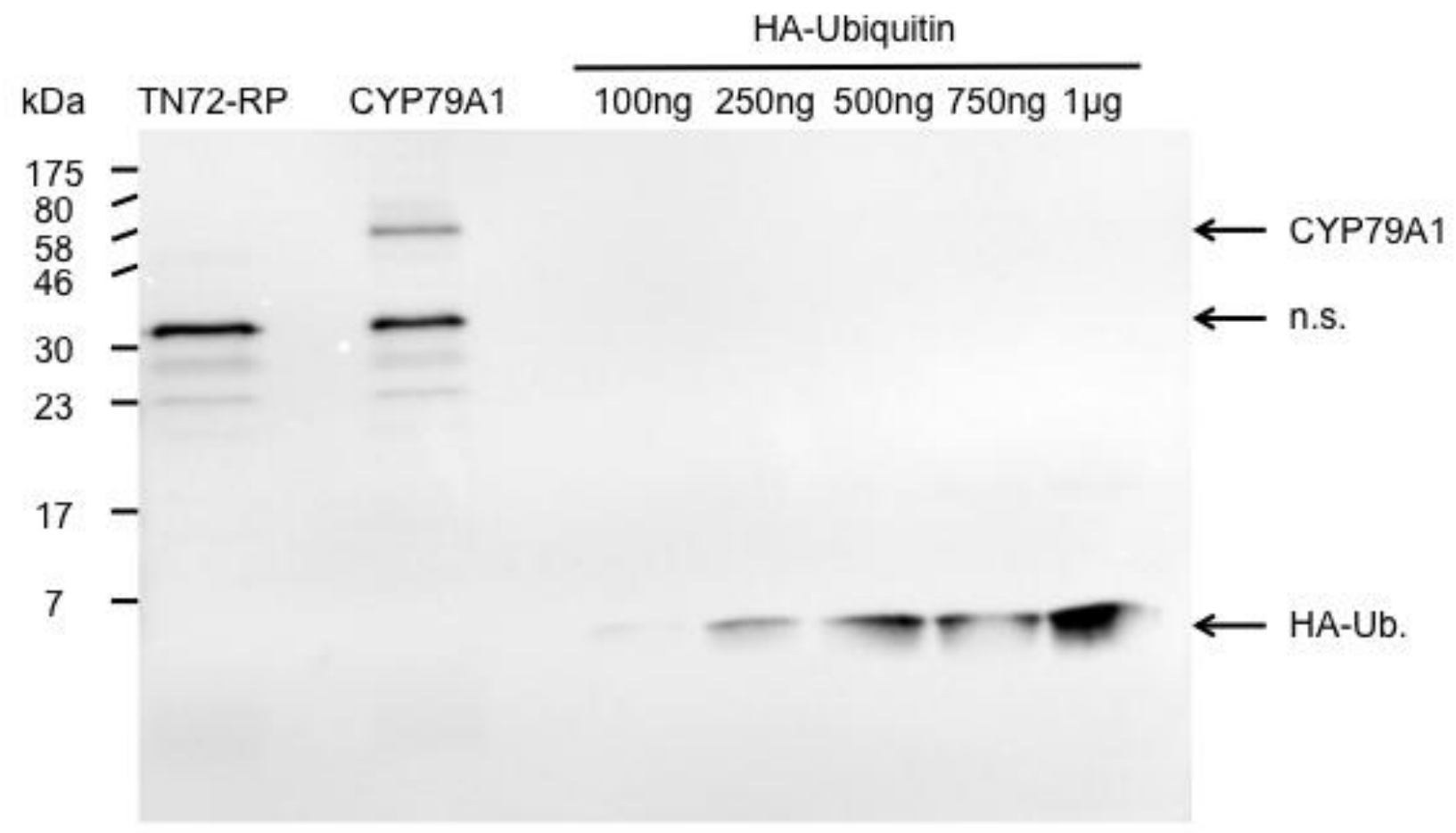

Figure 4 




Figure 5 


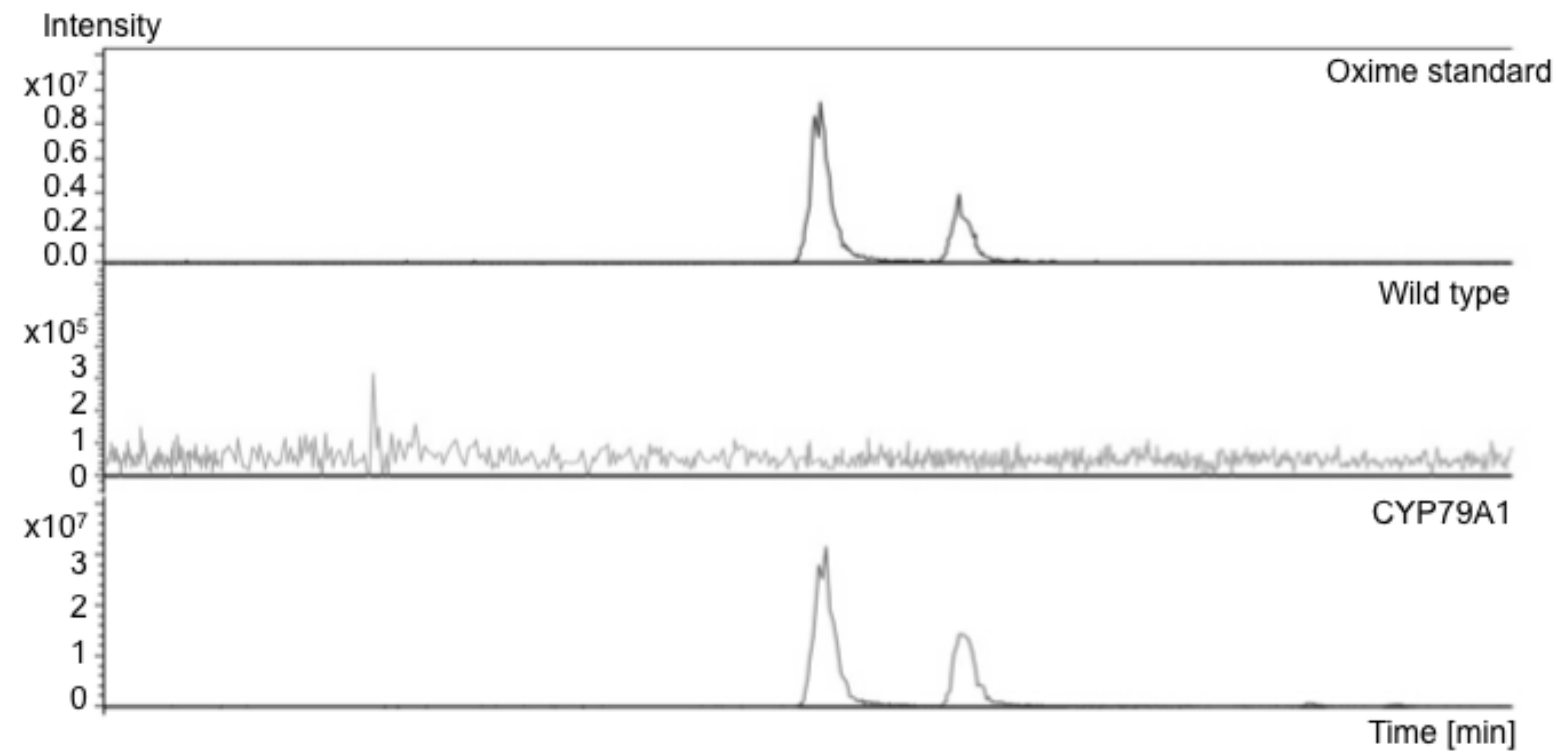

Figure 6 